\title{
BMJ Open What are the associations between neighbourhood walkability and sedentary time in New Zealand adults? The URBAN cross-sectional study
}

\author{
Erica Hinckson, ${ }^{1}$ Ester Cerin, ${ }^{2,3}$ Surzanne Mavoa, ${ }^{4,5}$ Melody Smith, ${ }^{6}$ \\ Hannah Badland, ${ }^{7}$ Karen Witten, ${ }^{5}$ Robin Kearns, ${ }^{8}$ Grant Schofield ${ }^{1}$
}

To cite: Hinckson E, Cerin E, Mavoa S, et al. What are the associations between neighbourhood walkability and sedentary time in New Zealand adults? The URBAN crosssectional study. BMJ Open 2017;7:e016128. doi:10.1136/ bmjopen-2017-016128

- Prepublication history for this paper is available online. To view these files please visit the journal online (http://dx.doi. org/10.1136/bmjopen-2017016128).

Received 1 February 2017 Revised 2 July 2017 Accepted 14 August 2017
CrossMark

For numbered affiliations see end of article.

Correspondence to Professor Erica Hinckson; erica.hinckson@aut.ac.nz

\section{ABSTRACT}

Objectives We estimated associations between objectively determined neighbourhood 'walkability' attributes and accelerometer-derived sedentary time (ST) by sex, city or type of day.

Design A cross-sectional study.

Setting The URBAN (Understanding the Relationship between Activity and Neighbourhoods) study was conducted in 48 neighbourhoods across four cities in New Zealand (August 2008 to October 2010).

Participants The response rate was 41\% (2029 recruited participants/5007 eligible households approached). In total, 1762 participants (aged $41.4 \pm 12.1$, mean \pm SD) met the data inclusion criteria and were included in analyses. Primary and secondary outcome measures The exposure variables were geographical information system (GIS) measures of neighbourhood walkability (ie, street connectivity, residential density, land-use mix, retail footprint area ratio) for street network buffers of $500 \mathrm{~m}$ and $1000 \mathrm{~m}$ around residential addresses. Participants wore an accelerometer for 7 days. The outcome measure was average daily minutes of ST.

Results Data were available from 1762 participants (aged $41.4 \pm 12.1$ years; $58 \%$ women). No significant main effects of GIS-based neighbourhood walkability measures were found with ST. Retail footprint area ratio was negatively associated with sedentary time in women, significant only for $500 \mathrm{~m}$ residential buffers. An increase of 1 decile in street connectivity was significantly associated with a decrease of over 5 min of ST per day in Christchurch residents for both residential buffers.

Conclusion Neighbourhoods with proximal retail and higher street connectivity seem to be associated with less ST. These effects were sex and city specific.

\section{BACKGROUND}

Sedentary behaviour is a common behaviour in adults, characterised by sitting times (median or mean) of 5 to 8 hours per day. ${ }^{12}$ Evidence indicates that prolonged periods of inactivity, induced through sitting, have detrimental health effects. ${ }^{3}$ A dose-response association has been reported between sitting time and all-cause mortality and cardiovascular

\section{Strengths and limitations of this study}

- The strength of this paper is the large sample size from across four cities in New Zealand with sufficient numbers to make intercity comparisons and the use of objective measures for sedentary time and the built environment.

- Data on retail floor area were not available and so the retail building footprint was used as a proxy.

- The retail land use was derived from zoning data, which potentially excludes smaller retail areas.

- The effects of neighbourhood self-selection and neighbourhood characteristics that promoted sedentary time were not differentiated.

- The lack of variation between New Zealand cities (low walkability in the global sense) may have also influenced our findings.

disease,${ }^{34}$ as well as higher risk for obesity and type 2 diabetes. ${ }^{5}$ These adverse effects are present even in individuals who meet public health guidelines for moderate to vigorous physical activity. ${ }^{6}$

Correlates of sedentary behaviour identified thus far in adults include age, attitudes, body mass index, depressive symptoms/ quality of life, education, employment status, gender, income, moderate to vigorous physical activity and smoking status. ${ }^{7}$ A socioecological approach to understanding health behaviours suggests that broader factors such as the built environment may play a role. ${ }^{8}$ However, while there is a large body of research focusing on behavioural and sociodemographic correlates of sedentary behaviour, research on social and environmental correlates remains scarce.

A recent systematic review on built environment attributes and sedentary behaviour relationships ${ }^{8}$ found that very few studies showed significant associations between neighbourhood built environment attributes and 
sedentary behaviours in the expected direction. However, overall, residents living in urban areas with diverse destinations close to their residential address had lower levels of sedentary behaviour. ${ }^{8}$ It is theorised that environments more conducive to physical activity may encourage less sitting. ${ }^{9}$ It is also theorised that the neighbourhood environment may have an added direct influence on women than men because, in general, women are more likely to engage with their residential neighbourhood through shopping/running errands and therefore more likely to be affected by the neighbourhood design. ${ }^{10}$

Studies thus far have used a mixture of self-reported and objectively measured sedentary behaviour and built environment measures. The latest review by Kooshari and colleagues ${ }^{8}$ did not differentiate between objective and subjective measures of either the built environment or sedentary behaviour, presumably due to the limited number of studies available in this area. Objective measures are generally more reliable and valid than self-reports. ${ }^{11}$

In response, the main aim of this paper was to examine associations between geographical information system (GIS)-measured components of the neighbourhood built environment within $500 \mathrm{~m}$ and $1000 \mathrm{~m}$ individual buffer zones of participant's homes and objectively measured sedentary time. We also explored whether these associations varied by sex, city or type of day (weekday vs weekend).

\section{METHODS}

Data for the current analysis were supplied by the Understanding the Relationship between Activity and Neighbourhoods (URBAN) study. A detailed description of the methods has been reported previously. ${ }^{12}$ Briefly, cross-sectional data were collected from designated neighbourhoods in four cities in New Zealand: North Shore, Waitakere, Wellington and Christchurch. The URBAN Study commenced in April 2008 in North Shore City and was completed in August 2010 in Christchurch. Although data collection started at different time points across the four locations, they were collected in a balanced manner across 12-14 months in each location to avoid clustering of seasonal effects by city. The host institutions of the research granted ethical approval for the study procedures (AUTEC: 07/126, MUHECN: 07/045).

\section{Neighbourhood selection}

Twelve neighbourhoods were selected from each city, resulting in a total of 48 neighbourhoods being sampled. Neighbourhoods were selected based on the walkability profile and ethnic population distribution (Māori, indigenous people) within contiguous mesh blocks (geographic census unit of approximately 100 households, median area: $0.30 \mathrm{~km}^{2}$, range: $1.03 \mathrm{~km}^{2}$ ) of the selected cities. ${ }^{13}$ Neighbourhood selection resulted in three high walkability/high Māori, three high walkability/low Māori, three low walkability/high Māori and three low walkability/low
Māori neighbourhoods. The study was relevant nationally by oversampling for Māori. Māori are the indigenous people of New Zealand and are the second largest ethnic group (after New Zealand European) in New Zealand. ${ }^{14}$ The walkability index was based on measures of street connectivity, net dwelling density, land-use mix and retail footprint area ratio, and was generated using GIS software, ArcInfo V.9.1 (Environmental Systems Research Institute, Redlands, California, USA). The construction of these measures replicated existing research. ${ }^{15}$

\section{Procedures}

The method of recruitment was by door to door; random start points were created in each neighbourhood and every $\mathrm{n}^{\text {th }}$ house ( $\mathrm{n}$ ranged from one to four according to neighbourhood net dwelling density) was approached. Five maximum visits were made to the eligible house. One adult (20-65 years) per household was invited to participate. Once the participant agreed to take part in the study, two subsequent visits were arranged 8 days apart. At the first visit, the study was introduced, informed consent was gained, and the accelerometer and compliance $\log$ were provided to the participants. A compliance $\log$ is a 'diary' where participants note the time they have taken off or put on the accelerometer and reason, time they went to bed or woke up in the morning or any other information relevant to the accelerometer. At the second visit, the researcher collected the accelerometer and compliance log (participants wore the accelerometer for 7 consecutive days) and completed the survey and anthropometric measures.

From each neighbourhood, 42 adults were randomly recruited. Participants from selected households were identified using the next birthday method. Exclusion criteria were falling outside the age ranges, not intending to live in the household over the measurement period, unable to speak the English language or impaired ability to walk.

\section{Measures}

Sedentary time

Sedentary time was objectively measured with the Actical accelerometer (Mini-Mitter, Sunriver, Oregon, USA) fitted to an elastic waistband and worn above the right hip. The units have been shown to be reliable and valid. ${ }^{16}{ }^{17}$ Prior to distribution, the units were tested for functionality and set up to record physical activity in $30 \mathrm{~s}$ epochs. Participants were instructed to wear the monitors during waking hours, but remove them when participating in water-based or contact activities. The threshold for sedentary time was set to $<100$ counts/minute using the Crouter 2R equation for the count threshold. ${ }^{18}$ Data from participants with at least 10 hours of wear time for at least 5 days (including 1 weekend day) were included in the analyses. Non-wear time was defined as $60 \mathrm{~min}$ or more of consecutive zero counts. ${ }^{19}{ }^{20}$ Daily measures of sedentary time were aggregated by averaging across all days for each participant. 


\section{Demographic variables}

Participants completed a face-to-face survey from which the following demographic characteristics were extracted: age, sex, ethnicity, marital status, academic qualification, total household income and employment status.

\section{Walkability measures}

We extracted measures of neighbourhood built environment from a GIS database that used ArcGIS V.9.3 software (Environmental Systems Research Institute). The methods have been described in detail elsewhere. ${ }^{12} 21$ Briefly, the walkability variables were:

- Street connectivity, estimated by calculating intersection density, the number of intersections per square kilometre. Intersections were extracted from road centreline data provided by Territorial Local Authorities.

- Net dwelling density, number of dwellings, estimated by dividing the number of dwellings by the residential land area for each mesh block. Dwelling data were sourced from the 2006 New Zealand census.

- Mixed land use, use of land including commercial, residential, industrial, open space and other, was calculated using an entropy index, ${ }^{15}$ where 0 indicates homogeneity of land use and 1 heterogeneity. Zoning data sourced from Territorial Local Authorities were used as a proxy for land use.

$\mathrm{LUM}=-\frac{\sum_{\mathrm{k}}\left(\mathrm{p}_{\mathrm{k}} \ln \mathrm{p}_{\mathrm{k}}\right)}{\ln \mathrm{N}}$ where $k$ is the land use category, $\mathrm{p}$ is the proportion of land area in a specific land use and $N$ is the number of land uses.

- Retail footprint area ratio, which, due to the lack of floor area data, was estimated as the area of the retail building footprint divided by the area of the retail land parcel. Participants with no retail land use in their buffer were assigned a retail footprint area ratio of zero. A higher ratio facilitates pedestrian access as it has smaller setbacks and less surface parking. ${ }^{22}$

Walkability measures were calculated for administrative units and also for $500 \mathrm{~m}$ and $1000 \mathrm{~m}$ road network buffers calculated around participants' residential addresses. Road network buffers were created using the ArcGIS Service Area function. Road centreline data were sourced from Territorial Local Authorities, and prior to calculation of the buffers, roads not accessible to pedestrians (eg, motorways and on-ramps and off-ramps) were removed.

\section{Data analytic plan}

Data were analysed in July 2014. Descriptive statistics (means, SDs and percentages) were computed for the whole sample and by city for all variables. Generalised additive mixed models $(\mathrm{GAMMs})^{23}$ were used to estimate associations between walkability and sedentary time. GAMMs can account for dependency in residuals (multiple measures taken on the same participants sampled from selected administrative units) and estimate complex, dose-response relationships of unknown form. $^{23}$ Three-level random intercept GAMMs with
Gaussian variance and identity link functions, appropriate for approximately normally distributed residuals (diagnostic tests were conducted to determine the appropriateness of the variance and link functions), were used to estimate associations between walkability and sedentary time. These models accounted for three levels of variability in the outcome-namely, variability at the administrative units selected for participant recruitment, at the person level (ie, between-participant differences) and at the within-person level (within-participant differences between weekday and weekend day estimates of sedentary time).

First, a GAMM estimated the independent associations of sociodemographic characteristics, type of day and study sites with accelerometry-based sedentary time. Then, separate main-effect GAMMs estimated the dose-response relationships of walkability components for the $500 \mathrm{~m}$ and $1 \mathrm{~km}$ road network buffers, respectively, with accelerometry-based sedentary time, adjusting for city, sociodemographic covariates (sex, age, marital status, educational attainment, ethnicity and employment status), neighbourhood deprivation (NZ Deprivation Index), average minutes of accelerometer wear time, number of valid days of wear and type of day (weekday vs weekend day). It was possible to simultaneously enter all GIS variables in the GAMMs as they were not collinear (mean absolute correlation $=0.26$; range of absolute correlations $=0.07-0.48)$. It was possible to simultaneously enter all GIS variables in the GAMMs as they were not collinear (mean absolute correlation $=0.20$; range of absolute correlations $=0.07-0.48$; mean variance inflation factor, $\mathrm{VIF}=1.14$; range of $\mathrm{VIF}=1.01-1.56$ ). Curvilinear relationships of environmental attributes with outcomes were estimated using non-parametric smooth terms in GAMMs, which were modelled using thin-plate splines. ${ }^{23}$ Smooth terms failing to provide sufficient evidence of a curvilinear relationship (based on Akaike Information Criterion (AIC)) were replaced by simpler linear terms. Separate GAMMs were run to estimate two-way and three-way walkability component by sex, city and type of day interaction effects. The significance of interaction effects was evaluated by comparing AIC values of models with and without a specific interaction term. An interaction effect was deemed significant if it yielded a $>2$ unit smaller AIC than the main effect model. ${ }^{24}$ Significant interaction effects were probed by computing the sex-specific, city-specific and/or type-of-day-specific associations (as appropriate) of GIS-based components of walkability with accelerometry-based sedentary time via linear functions.

There were $12.5 \%$ cases with missing or invalid accelerometer data (252 out of 2014), and these were excluded in the analyses. Participants with valid accelerometer data were older $(\mathrm{p}<0.001)$, more likely to live in high socioeconomic status neighbourhoods $(p=0.010)$ and be employed $(\mathrm{p}<0.001)$. All regression models were adjusted for these variables. There were less than $1.3 \%$ of remaining participants with missing data on any other 


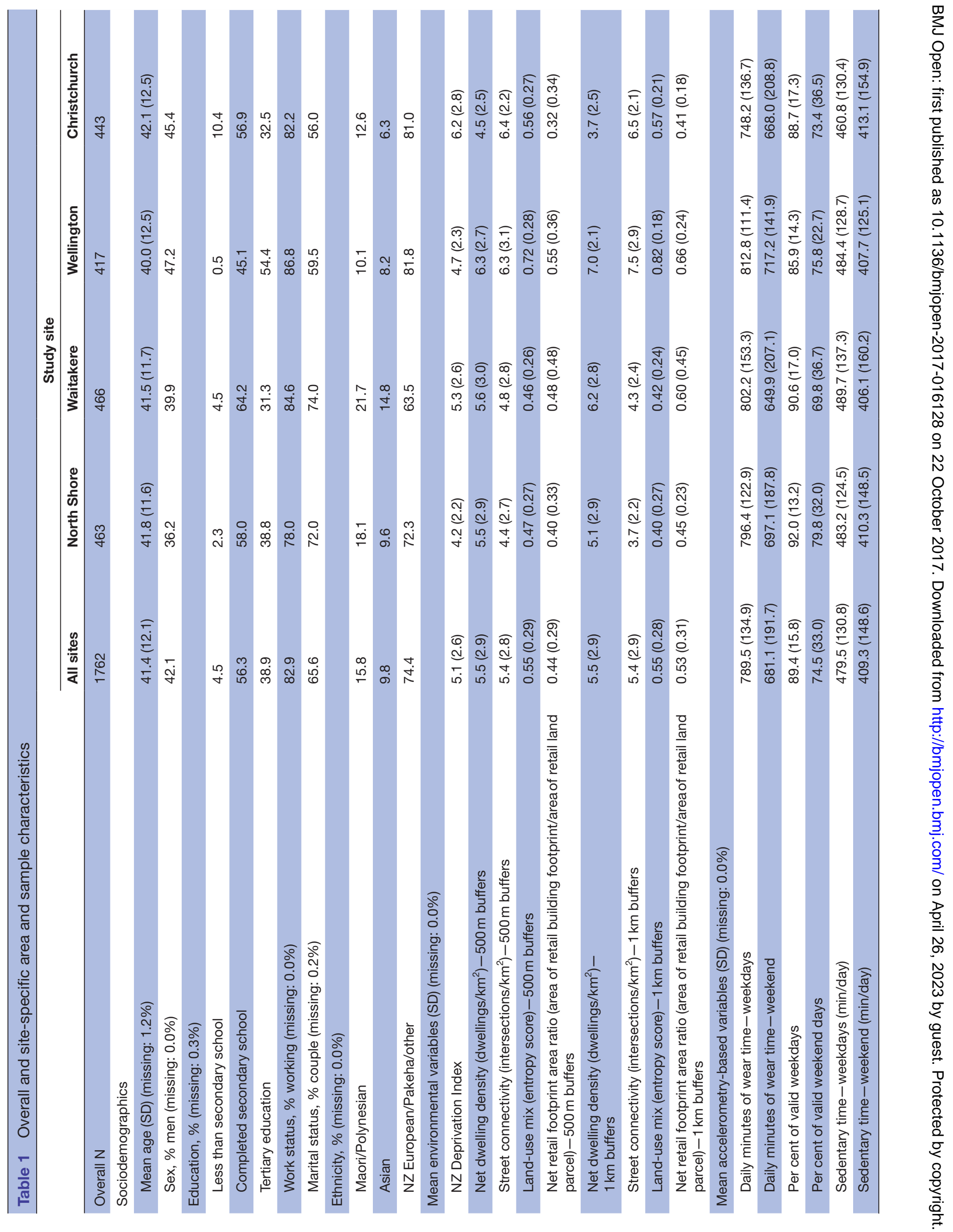


Table 2 Associations of sociodemographic characteristics, type of day and study sites with accelerometry-based sedentary time

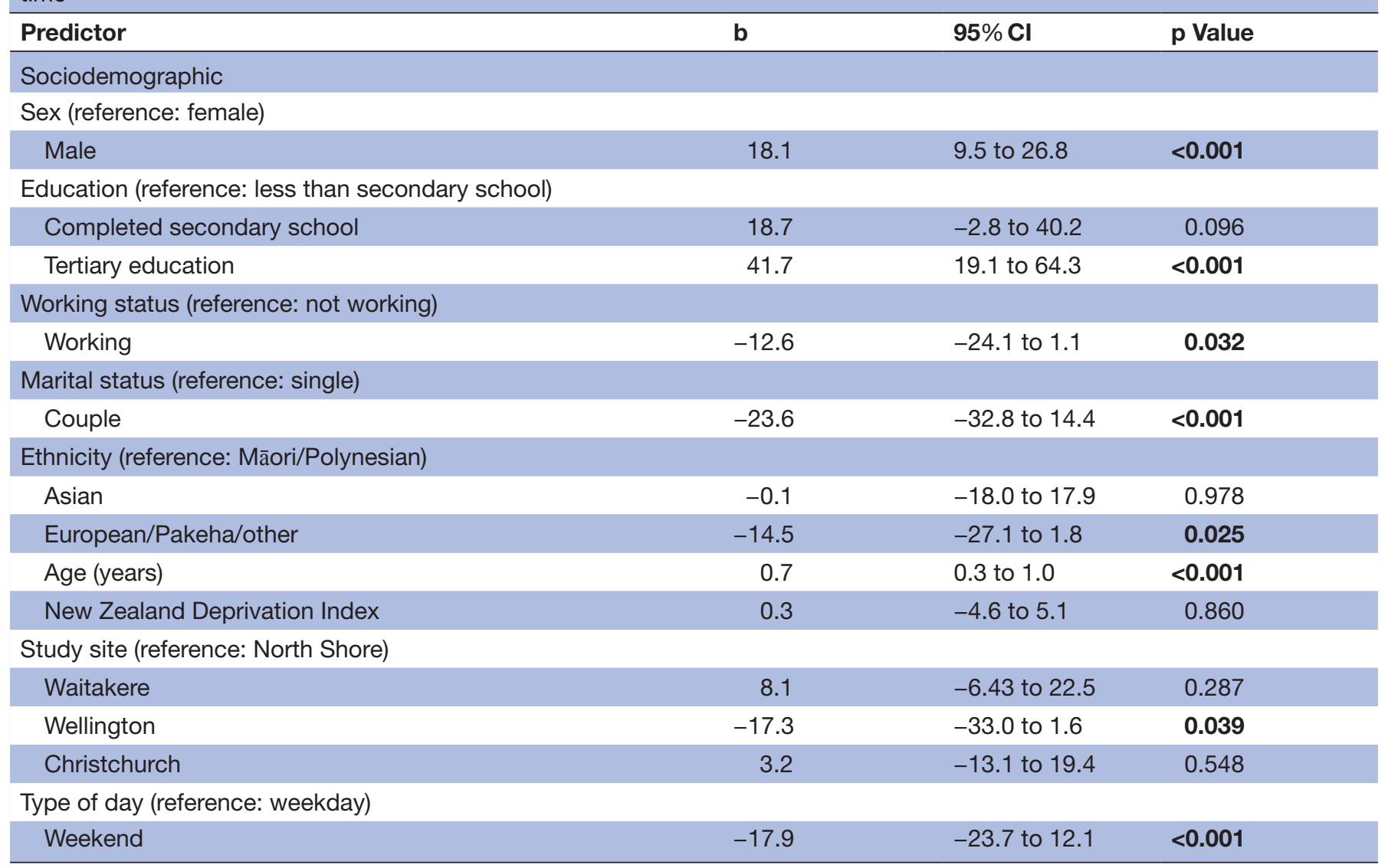

The regression model was adjusted for valid days of accelerometer wear and average daily minutes of wear and accounted for correlated residuals at the neighbourhood and participant levels. b, regression coefficients.

variables. Given the low percentage of missing data, these values were imputed with the most common values for the sample (mean values or modes, as appropriate). All analyses were conducted in $\mathrm{R}^{25}$ using the packages 'car',26 and 'mgcv'. 23

\section{Data availability}

The datasets generated during and/or analysed during the current study are not publicly available due to the institution's ethical approval process and the requirement of consent by participants (indigenous and otherwise) but are available from the corresponding author on reasonable request.

\section{RESULTS}

The response rate was $41 \%$ (2029 recruited participants / 5007 eligible households approached). In total, 1762 participants (aged $41.4 \pm 12.1$, mean \pm SD) met the data inclusion criteria and were included in analyses. Table 1 reports the descriptive statistics of participants with accelerometer data for each city. This includes sample sociodemographic characteristics, GIS-based walkability components and accelerometry-based variables. Mean age of the total sample was 41.4 years $(\mathrm{SD}=12.1)$. Average sitting time was lower on weekends (mean=409 $\mathrm{min} /$ day) than weekdays (mean=480 $\mathrm{min} /$ day). Participants living in Christchurch had the highest sitting time on weekends, but the lowest on weekdays.

On average, selected neighbourhoods in Christchurch had the lowest levels of net dwelling density and retail footprint area ratios, while Wellington had the highest levels of net dwelling density, intersection density and land-use mix.

Associations between sociodemographic variables, city and type of day with accelerometry-based sedentary time

Men, older participants and more highly educated participants engaged in significantly more sedentary time than their counterparts (table 2). For example, compared with participants with less than secondary schooling, those who completed tertiary education accumulated 41.7 more minutes of sedentary time per day. Being employed, living with a partner (as opposed to being single) and not being of Asian or Māori/Polynesian ethnicity were associated with lower levels of sedentary time. On average, participants from Wellington accumulated less sedentary time than those from other study sites. Weekend days were on average associated with approximately 18 fewer minutes of sedentary time than weekdays (table 2 ). 
Table 3 Associations of GIS-based walkability components for $500 \mathrm{~m}$ and $1 \mathrm{~km}$ residential buffers with accelerometry-based sedentary time

\begin{tabular}{|c|c|c|c|c|c|c|}
\hline \multirow[b]{2}{*}{ Predictor } & \multicolumn{3}{|c|}{$500 \mathrm{~m}$ residential buffers } & \multicolumn{3}{|c|}{$1 \mathrm{~km}$ residential buffers } \\
\hline & b & $95 \% \mathrm{Cl}$ & $\mathrm{p}$ Value & b & $95 \% \mathrm{Cl}$ & p Value \\
\hline \multicolumn{7}{|l|}{ Main effect models } \\
\hline Net dwelling density & -0.39 & -2.08 to 1.31 & 0.654 & -0.57 & -2.49 to 1.35 & 0.564 \\
\hline Street connectivity & 0.14 & -1.90 to 2.18 & 0.892 & -0.34 & -2.58 to 1.90 & 0.768 \\
\hline Land-use mix* & 1.04 & -1.64 to 1.15 & 0.265 & 1.03 & -1.08 to 3.14 & 0.339 \\
\hline Net retail footprint area ratio* & -0.24 & -1.64 to 1.15 & 0.733 & -0.02 & -1.88 to 1.84 & 0.982 \\
\hline \multicolumn{7}{|l|}{ Interaction effects } \\
\hline \multicolumn{7}{|l|}{ Street connectivity by study site } \\
\hline Effect of street connectivity in North Shore & 1.02 & -2.37 to 4.43 & 0.556 & 0.20 & -4.00 to 4.41 & 0.927 \\
\hline Effect of street connectivity in Waitakere & 1.26 & -2.02 to 4.55 & 0.452 & -0.65 & -4.43 to 3.12 & 0.733 \\
\hline Effect of street connectivity in Wellington & 1.55 & -1.78 to 4.88 & 0.363 & 2.12 & -1.14 to 5.38 & 0.203 \\
\hline Effect of street connectivity in Christchurch & -5.06 & -9.25 to 0.86 & 0.019 & -5.18 & -9.50 to 0.86 & 0.019 \\
\hline \multicolumn{7}{|l|}{ Net retail footprint area (NRFA) ratio by sex } \\
\hline Effect of NRFA in men & 1.49 & -0.36 to 3.35 & 0.116 & 1.58 & -0.86 to 4.01 & 0.205 \\
\hline Effect of NRFA in women & -1.95 & -3.60 to 0.29 & 0.022 & -1.48 & -3.59 to 0.64 & 0.172 \\
\hline
\end{tabular}

All models adjusted for other walkability components, sociodemographics, New Zealand Deprivation Index, study site and type of day (weekday vs weekend). All models accounted for correlated residuals at the neighbourhood and participant levels. b, regression coefficients. ${ }^{*}$ The original values of land-use mix and net retail footprint area ratio were multiplied by 10 to obtain point estimates of the regression coefficients that would correspond to the difference in minutes/day of sedentary time associated with a 0.10 increase in these environmental predictors rather than the difference in outcomes between the theoretical minimum (ie, 0 ) and maximum (ie, 1) values of the predictors.

\section{Associations of walkability components with accelerometry- based sedentary time}

No significant main effects of GIS-based walkability components with accelerometry-based sedentary time were found (table 3). However, significant interaction effects were found between street connectivity by city and retail footprint area ratio by sex. Street connectivity was significantly negatively associated with sedentary time in Christchurch only. Among participants from Christchurch, an increase of 1 decile in street connectivity was associated with a decrease of over 5 min of sedentary time per day. This effect was observed for both residential buffer sizes. While retail footprint area ratio was not predictive of sedentary time in men, it was negatively associated with sedentary time in women across all cities. However, this effect was stronger (and significant only) for $500 \mathrm{~m}$ residential buffers across the four cities (table 3 ).

The proportions of sedentary time variance attributable to between-neighbourhood, between-participant (within neighbourhoods) and between-day (within participants) differences were $0.02(p=0.002), 0.35(p<0.001)$ and 0.63 $(\mathrm{p}<0.001)$, respectively. The final GAMMs, including all covariates, environmental variables and interaction terms, explained $95 \%$ of the between-neighbourhood, $34 \%$ of between-participant and $55 \%$ of between-day variance. The proportions of unexplained sitting time variance attributable to differences between neighbourhood, persons and days were approximately $<0.01 \quad(\mathrm{p}>0.250)$, $0.45(\mathrm{p}<0.001)$ and $0.54(\mathrm{p}<0.001)$, respectively.

\section{DISCUSSION}

There were no significant main effects of GIS-based walkability on sedentary time; however, significant interaction effects were found between street connectivity in one city (Christchurch) and retail footprint area ratio in women. This and the study by Van Dyck ${ }^{19}$ and colleagues are the only studies that have examined associations between objectively measured built environment and sedentary time in adults.

Our a priori assumption was that built environments more conducive to physical activity may encourage less time spent in sedentary behaviour. Our findings do not necessarily support this, as there were no significant main effects of GIS-based walkability on sedentary time and only $2 \%$ of the variance in sitting time was due to differences between neighbourhoods. Perhaps, sedentary behaviour is influenced by neighbourhood cultural or social phenomena or built environment factors not measured in the study such as public transport (Wellington region has the highest use of public transport per capita use in New Zealand). Also, the administrative units used to capture neighbourhood-level variability in sedentary time may not represent the optimal geographical scale to detect environmental influences on this specific behaviour. Moreover, since sedentary behaviour is not the reverse of physical activity, ${ }^{27}$ the presence of walkable features may not influence the transition from sitting to walking. Another explanation may be that since sedentary time seems to 
be associated with home-based activities (eg, television watching, computer use), ${ }^{28}$ perhaps built environment associations around smaller buffers (close proximity to the home) may have been more appropriate. Certainly, in our study, there was only one significant interaction effect observed within a $1000 \mathrm{~m}$ buffer and only two interaction effects within the $500 \mathrm{~m}$ buffer. Nonetheless, smaller buffers may be problematic in countries in which neighbourhoods are of comparatively low walkability such as New Zealand. ${ }^{29}$ On the other hand, a measure of regional accessibility may need to be included to account for environments of residents that commonly access (work and non-work) beyond the neighbourhood. Regional accessibility is defined as being determined by large regional shopping centres and employment clusters, usually farther away, offering a variety of services. ${ }^{30}$ It is possible that multipurpose trips are done in this way, conceivably reducing the possibility of local accessibility irrespective of the neighbourhood environment. Ubiquity of car ownership, as is the case for New Zealand, is also important. Ivory and colleagues $^{10}$ saw a strong relationship between built environment characteristics and car access, where almost half of those with restricted car access lived in the most connected street network areas, compared with approximately one-third who had full car access. Lastly, it is plausible that different GIS measures may need to be considered for the development of sedentary behaviour index in the future, but extensive conceptualisation and research must take place first.

In the interaction effects model, two relationships showed significance: street connectivity in Christchurch (within both $500 \mathrm{~m}$ and $1000 \mathrm{~m}$ neighbourhood buffer) and retail footprint area ratio in women (within $500 \mathrm{~m}$ neighbourhood buffer only). For participants from Christchurch, an increase of 1 decile in street connectivity was associated with a decrease of over $5 \mathrm{~min}$ of sedentary time per day. The result makes sense due to the city's relatively flat topography and city design in comparison to Auckland and Wellington. Christchurch was one of four cities in the world (prior to the 2010 Christchurch earthquake) that was designed in a rectangular grid fashion with a central city square, surrounded by four city squares and a parklands area. ${ }^{31}$ Literature has shown that street connectivity is positively associated with overall walking ${ }^{32}$ and walking for transport. ${ }^{32}{ }^{33}$ In terms of the second interaction effect, a significant association was observed between sedentary time and higher footprint area retail ratio within the $500 \mathrm{~m}$, but not the $1000 \mathrm{~m}$ neighbourhood buffer. Across all cities, the floor area retail ratio within a $500 \mathrm{~m}$ neighbourhood buffer was predictive of less sedentary time in women but not men. Aside from the sex difference, we theorised that neighbourhoods more conducive to physical activity, in this case presence of retail (any type) in close proximity, may encourage reduction of sedentary time. Mixing residential neighbourhoods with retail and other uses is not a new concept and remained the design of traditional European cities and towns for several centuries. Traditional neighbourhoods morphed around the individual's needs to walk short distances to a destination. In terms of the sex difference, a similar sex association has been shown with self-reported sedentary behaviour in women and neighbourhood walkability ${ }^{34}$ : women residing in low-walkable neighbourhoods reported increased levels of television viewing compared with those who lived in higher-walkable neighbourhoods. ${ }^{34}$ While other studies have not investigated sex differences, Kooshari and colleagues ${ }^{35}$ reported that in high-walkable Australian neighbourhoods, in particular those with high retail footprint area ratio, residents spent less time sitting in cars, while in Belgian adults, Van Dyck and associates ${ }^{19}$ found that daily sedentary time was higher for residents living in a high-walkable environment. Nonetheless, according to our results, one may infer that women who reside in neighbourhoods with better access to retail spend less time in sedentary pursuits because they walk to a destination of interest frequently. These associations need to be investigated further to understand the reasons behind these.

The strength of this paper is the large sample size from across four cities in New Zealand with sufficient numbers to make intercity comparisons. An additional strength is the use of objective measures for sedentary time and the built environment. Notwithstanding the strengths of the study, there are limitations that need to be acknowledged. One of the main limitations is that the data were derived from a cross-sectional study and causation cannot be assumed. While the retail footprint area ratio potentially identifies differences between big-box retail and strips (includes car parking), the spatial data used for this measure were limited in two ways. First, data on retail floor area ratio were not available and so the retail building footprint was used as a proxy. Second, retail land use was derived from zoning data, which potentially excludes smaller retail areas and does not necessarily correspond to current use. Further, given that our study neighbourhoods were located in areas unlikely to be within $1000 \mathrm{~m}$ of big-box retail, in our study we consider the retail footprint area ratio as a proxy for access to retail. We were unable to differentiate between the effects of neighbourhood self-selection and neighbourhood characteristics that promoted sedentary time. It is possible that an alternative aggregate of GIS measures might have captured city differences better than the measures that we have used; for example, topography was not included in the GIS variables measured. The lack of variation between New Zealand cities (low walkability in the global sense) may have also influenced our findings and possibly the reason for the difference in findings with the Belgian study. ${ }^{19}$ Since we used accelerometry to determine sedentary behaviour, we were unable to determine the type of sedentary behaviour participants engaged in. Finally, hip-mounted accelerometers can misclassify very light activity and standing with sedentary behaviour. For 
this reason, hip-mounted accelerometers tend to overestimate sedentary behaviour.

\section{CONCLUSION}

Our results imply that sedentary behaviour (time) was not correlated with the built environment in the New Zealand context. While there were no significant main effects between GIS-based walkability and sedentary time, neighbourhoods within a $500 \mathrm{~m}$ neighbourhood buffer that included retail were associated with less sedentary time in women residents only. In Christchurch, higher street connectivity was associated with less sedentary time. Taken together, it is possible that since sedentary behaviour is not the reverse of physical activity, ${ }^{27}$ the presence of walkable features may not influence the transition from sitting to walking and other factors (social and cultural) may supersede. This paper provided several alternatives to explain these results.

\section{Author affiliations}

${ }^{1}$ Human Potential Centre, Auckland University of Technology, Auckland, New Zealand ${ }^{2}$ Institute for Health and Ageing, Australian Catholic University, Melbourne, Victoria, Australia

${ }^{3}$ School of Public Health, The University of Hong Kong, Hong Kong, China ${ }^{4}$ McCaughey VicHealth Community Wellbeing Unit, Faculty of Medicine, Dentistry and Health Science, Melbourne School of Population and Global Health, , University of Melbourne, Melbourne, Victoria, Australia

${ }^{5}$ SHORE \& Whariki Research Centre, Massey University, Auckland, New Zealand

${ }^{6}$ School of Nursing, The University of Auckland, Auckland, New Zealand ${ }^{7}$ Centre for Urban Research, RMIT University, Melbourne, Victoria, Australia ${ }^{8}$ School of Environment, Faculty of Science, The University of Auckland, Auckland, New Zealand

Acknowledgements The authors gratefully acknowledge the participants who completed the study, research assistants who collected the data and the territorial authorities for providing the GIS datasets.

Contributors EH has provided the first draft and subsequent drafts, is the corresponding author and conceptualised the content of manuscript and analysis. $\mathrm{EC}$ analysed the data and provided the results and tables. SM conducted the GIS analysis and contributed to the manuscript. MS contributed to the manuscript. HB managed the study and contributed to the manuscript. KW is coprimary investigator and contributed to the manuscript. RK contributed to the manuscript. GS is coprimary investigator and contributed to the manuscript.

Funding This work was funded by the Health Research Council (HRC) of New Zealand (grant nos 07/356 and 08/048). EC is supported by an Australian Research Council (ARC) Future Fellowship (FT no 140100085). HB and SM are in part supported by VicHealth, the National Health and Medical Research Council (NHMRC) Centre of Research Excellence in Healthy Liveable Communities (no 1061404) and The Australian Prevention Partnership Centre (no 9100001) with funding provided by NHMRC, ACT Health, NSW Health, the Australian National Preventive Health Agency, the Hospitals Contribution Fund of Australia and the HCF Research Foundation.

Competing interests None declared.

Patient consent Obtained.

Ethics approval Auckland University of Technology (AUTEC: 07/126) and Massey University (MUHECN: 07/045) ethics committees.

Provenance and peer review Not commissioned; externally peer reviewed.

Data sharing statement The datasets used and/or analysed during the current study are available from the corresponding author on reasonable request.

Open Access This is an Open Access article distributed in accordance with the Creative Commons Attribution Non Commercial (CC BY-NC 4.0) license, which permits others to distribute, remix, adapt, build upon this work non-commercially, and license their derivative works on different terms, provided the original work is properly cited and the use is non-commercial. See: http://creativecommons.org/ licenses/by-nc/4.0/

(c) Article author(s) (or their employer(s) unless otherwise stated in the text of the article) 2017. All rights reserved. No commercial use is permitted unless otherwise expressly granted.

\section{REFERENCES}

1. Bauman A, Ainsworth BE, Sallis JF, et al. The descriptive epidemiology of sitting. A 20-country comparison using the International Physical Activity Questionnaire (IPAQ). Am J Prev Med 2011;41:228-35.

2. Hagströmer M, Troiano RP, Sjöström M, et al. Levels and patterns of objectively assessed physical activity - a comparison between Sweden and the United States. Am J Epidemiol 2010;171:1055-64.

3. Katzmarzyk PT, Church TS, Craig CL, et al. Sitting time and mortality from all causes, cardiovascular disease, and cancer. Med Sci Sports Exerc 2009;41:998-1005.

4. Thorp AA, Owen N, Neuhaus M, et al. Sedentary behaviors and subsequent health outcomes in adults a systematic review of longitudinal studies, 1996-2011. Am J Prev Med 2011;41:207-15.

5. Hu FB, Li TY, Colditz GA, et al. Television watching and other sedentary behaviors in relation to risk of obesity and type 2 diabetes mellitus in women. JAMA 2003;289:1785-91.

6. Healy GN, Wijndaele K, Dunstan DW, et al. Objectively measured sedentary time, physical activity, and metabolic risk. Diabetes Care 2008;31:369-71.

7. Rhodes RE, Mark RS, Temmel CP. Adult sedentary behavior: a systematic review. Am J Prev Med 2012;42:e3-28.

8. Koohsari MJ, Sugiyama T, Sahlqvist S, et al. Neighborhood environmental attributes and adults' sedentary behaviors: review and research agenda. Prev Med 2015;77:141-9.

9. Barnett A, Cerin E, Ching CS, et al. Neighbourhood environment, sitting time and motorised transport in older adults: a cross-sectional study in Hong Kong. BMJ Open 2015;5:e007557.

10. Ivory VC, Blakely T, Pearce J, et al. Could strength of exposure to the residential neighbourhood modify associations between walkability and physical activity? Soc Sci Med 2015;147:232-41.

11. Dyrstad SM, Hansen BH, Holme IM, et al. Comparison of selfreported versus accelerometer-measured physical activity. Med Sci Sports Exerc 2014;46:99-106.

12. Badland HM, Schofield GM, Witten $\mathrm{K}$, et al. Understanding the Relationship between Activity and Neighbourhoods (URBAN) Study: research design and methodology. BMC Public Health 2009;9:224.

13. Zealand SN. Census of population and dwellings: Auckland City. What we look like locally. Wellington: Statistics NZ, 2006.

14. Statistics New Zealand. New Zealand in profile: an overview of New Zealand's people, economy, and environment. Statistics New Zealand: Wellington, 2009.

15. Leslie E, Coffee N, Frank L, et al. Walkability of local communities: using geographic information systems to objectively assess relevant environmental attributes. Health Place 2007;13:111-22.

16. Esliger DW, Tremblay MS. Technical reliability assessment of three accelerometer models in a mechanical setup. Med Sci Sports Exerc 2006;38:2173-81.

17. Esliger DW, Probert A, Connor Gorber S, et al. Validity of the Actical accelerometer step-count function. Med Sci Sports Exerc 2007;39:1200-4.

18. Crouter SE, Dellavalle DM, Horton M, et al. Validity of the Actical for estimating free-living physical activity. Eur J Appl Physiol 2011;111:1381-9.

19. Van Dyck D, Cardon G, Deforche B, et al. Neighborhood walkability and sedentary time in Belgian adults. Am J Prev Med 2010;39:25-32.

20. Oliver M, Badland HM, Schofield GM, et al. Identification of accelerometer nonwear time and sedentary behavior. Res $Q$ Exerc Sport 2011;82:779-83.

21. Mavoa S, Witten K, Pearce J, et al. Measuring neighbourhood walkability in New Zealand cities, 2009.

22. Frank LD, Sallis JF, Saelens BE, et al. The development of a walkability index: application to the Neighborhood Quality of Life Study. Br J Sports Med 2010;44:924-33.

23. Wood S. Generalized additive models: an introduction with $R$. Boca Raton, FL: Chapman \& Hall, 2006.

24. Burnham K, Anderson D. Model selection and multimodel inference: a practical information-theoretic approach. 2nd edn. New York: Springer, 2002. 
25. Core Team R. R: a language and environment for statistical computing. Vienna, Austria: R Foundation for Statistical Computing, 2013.

26. Fox J, Weisberg S. An R companion to applied regression. 2nd edn. Thousand Oaks, CA: Sage, 2011.

27. Healy GN, Dunstan DW, Salmon J, et al. Television time and continuous metabolic risk in physically active adults. Med Sci Sports Exerc 2008;40:639-45.

28. Owen N, Sugiyama T, Eakin EE, et al. Adults' sedentary behavior determinants and interventions. Am J Prev Med 2011;41:189-96.

29. Sallis JF, Bowles HR, Bauman A, et al. Neighborhood environments and physical activity among adults in 11 countries. Am J Prev Med 2009;36:484-90.

30. Handy S. Regional versus local accessibility: implications for nonwork travel. Berkeley, CA: University of California Transportation Center, 1993:58-66.
31. Wilson J, Dowson S, Adam J, et al. Contextual historical overview of Christchurch City. Christchurch: Christchurch City Council, 2005.

32. Witten K, Blakely T, Bagheri N, et al. Neighborhood built environment and transport and leisure physical activity: findings using objective exposure and outcome measures in New Zealand. Environ Health Perspect 2012;120:971-7.

33. Koohsari MJ, Sugiyama T, Lamb KE, et al. Street connectivity and walking for transport: role of neighborhood destinations. Prev Med 2014;66:118-22.

34. Sugiyama T, Salmon J, Dunstan DW, et al. Neighborhood walkability and TV viewing time among Australian adults. Am J Prev Med 2007:33:444-9.

35. Koohsari MJ, Sugiyama T, Kaczynski AT, et al. Associations of leisure-time sitting in cars with neighborhood walkability. J Phys Act Health 2014;11:1129-32. 